\title{
Classroom Management of Mental Retardation
}

\author{
Bruno U.D. Onyekuru and Joyce NJOKU \\ Department of Educational Psychology, Guidance and Counselling \\ Faculty of Education, University of Port Harcourt \\ P.M.B 5323, Port Harcourt, Nigeria
}

Accepted: July 24, 2012 Published: September 12, 2012

Doi:10.5296/ijld.v2i5.2369 URL: http://dx.doi.org/10.5296/ijld.v2i5.2369

\begin{abstract}
Mental retardation is one of the major human development problems of our time. The consequences of mental retardation in the general population are alarming and devastating. Even the cost of managing the disorder cannot be met or borne by so many whose children are afflicted. Some people whose children are suffering from the disorder are handicapped and don't know what to do about it. It is based on this premise that the authors decided to bring this ugly trend to the awareness of many Nigerians especially school administrators. The paper started by examining the meaning of the disorder. The authors went ahead to enumerate the different classifications of mental retardation as well as the signs, symptoms and causes of this disorder. The paper ended by suggesting some practical ways the school administration and government can help in training these children thereby reducing the occurrence and prevalence of this disorder which is highly incurably by the present scientific knowledge.
\end{abstract}

Key words: $\quad$ Mental retardation, Classroom Management, Disability

\section{Introduction}

Mental Retardation (MR) is a generalized disorder appearing before 18 years particularly in early years of school life of the individual and it characterized by significantly impaired cognitive functioning and deficits in two or more adaptive behaviours (APA, 2005). Mental retardation is a condition that is present at birth and it is characterized by significantly below average intellectual functioning i.e. IQ is 70 or below (Halgin and Whitebour, 2005). It is a developmental disability that first appears in children under the age of 18. Mental retardation is defined as an intellectual functioning level (as measured by standard tests for intelligence quotient) that is well below average and significant limitations in daily living skills (adaptive functioning). Mental retardation varies in severity ranging from mild to profound. The diagnostic and Statistical Manual of Mental Disorders, (D S M-IV) is the diagnostic standard for mental healthcare professionals in the world over. The DSM-IV classifies four different degrees of mental retardation; mild, moderate, severe, and profound. These categories are based on the functioning level of the individual (Obidigbo, 2010). The authors noted that low intelligence quotient (IQ) scores and limitations in adaptive skills are the hallmarks of mental retardation. Aggression, self-injury, and mood disorders are sometimes associated with the disability. The severity of the symptoms and the age at which they first appear depend on the 
cause. Children who are mentally retarded reach developmental milestones significantly later than expected, if at all. If retardation is caused by chromosomal or other genetic disorders, it is often apparent from infancy. If retardation is caused by childhood illnesses or injuries, learning and adaptive skills that were once easy may suddenly become difficult or impossible to master. (Maduakonam, 1995). The authors added that a mentally retarded person is slow to learn and may be slow or limited in the development of physical skills. Additionally, physical handicaps may be present, such as speech impairments, visual impairments, hearing defects, or epilepsy. It has historically been defined as an intelligence quotient score under 70. Once focused almost entirely on cognition, the definition now includes both a component relating to mental functioning and one relating to individuals' functional skills in their environment. As a result, a person with below-average intelligence quotient (BAIQ) may not be considered mentally retarded unless there is deficit in adaptive functioning (APA, 2005). Syndromic mental retardation is intellectual deficits associated with other medical and behavioural signs and symptoms. Non-syndromic mental retardation refers to intellectual deficits that appear without their abnormalities. (Halgin \& Whitebourne, 2003; Durena \& Barlow, 2000).

The terms used to describe this condition are subject to a process called the euphemism treadmill because of the derogatory remarks attached to it. Such terms include idiot, moron, imbecile etc (Maduakonam, 1995; Obidigbo, 2005, 1995). This means that whatever term is chosen for this condition, it eventually becomes perceived as an insult. The terms mental retardation and mentally retarded were invented in the middle of the $20^{\text {th }}$ century to replace the previous set of terms, which were deemed to have become offensive. By the end of the $20^{\text {th }}$ century, these terms have come to be widely seen as disparaging and politically incorrect and in need of replacement.

Mental retardation affects about $2-3 \%$ of people. $75-90 \%$ of the affected people have mild retardation. Non-syndromic or idiopathic mental retardation accounts for 30-50\% cases. About a quarter of cases are caused by a genetic disorder (Durand \& Barlow, 2000).

\section{Signs and Symptoms}

The signs and symptoms of mental retardation are all behavioural. Most people with mental retardation do not look like they have any type of intellectual disability, especially if the disability is caused by environmental factors such as malnutrition or lead poisoning. The so-called "typical appearance" ascribed to people with mental retardation is only present in a minority of cases, all of which involve syndromic mental retardation.

Children with mental retardation may learn to sit up, to crawl, or to walk later than other children, or they may learn to talk later. Children with mental retardation may also exhibit some or all of the following characteristics:

- $\quad$ Delay in oral language development

- $\quad$ Deficits in memory skills

- $\quad$ Difficulty learning social rules

- $\quad$ Difficulty with problem solving skills

- Delay in the development of adaptive behaviours such as self-help or self-care skills

- $\quad$ Lack of social inhibitors.

Children with mental retardation learn more slowly than a typical child. Children may take longer to learn language, develop social skills, and take care of their personal needs, such as dressing or eating. Learning will take them longer, require more repetition, and skills may need to be adapted to their learning level. Nevertheless, virtually every child is able to learn, develop and become a participating member of the community. 
In early childhood, mild mental retardation (IQ 50-69, a cognitive ability about half to two-thirds of standard) may not be obvious, and may not be identified until children begin school. Even when poor academic performance is recognized, it may take expert assessment to distinguish mild mental retardation from learning disability or emotional/behavioural disorders. People with mild mental retardation are capable of learning, reading and handling some mathematics skills to approximately the level of a typical child aged 9 to 12 . They can learn self-care and practical skills, such as cooking or using the local mass transit system. As individuals with mild mental retardation reach adulthood, many learn to live independently and maintain gainful employment.

Moderate mental retardation (IQ 35-49) is nearly always apparent within the first years of life. Speech delays are particularly common signs of moderate mental retardation. People with moderate mental retardation need considerable supports in school, at home, and in the community in order to participate fully. While their academic potential is limited, they can learn simply health and safety skills and participate in simple activities. As adults, they may live with their parents, in a supportive group home, or even semi-independently with significant supportive services to help them, for example, manage their finances. As adults, they may work in a sheltered workshop. A person with severe or profound mental retardation will need more intensive support and supervision all over his or her entire life. They may learn some activities of daily living. Some will require full-time care by an attendant.

\section{Classification of Mental Retardation}

Mental retardation can come in any of these forms: Mild mental retardation, Moderate mental retardation, Severe mental retardation, Profound mental retardation.

3.1 Mild Mental Retardation: Mentally retarded population is in the mildly retarded category. Their intelligence quotient (IQ) score ranges from 50-75, and they can often acquire academic skills up to the $6^{\text {th }}$ grade level. They can become fairly self-sufficient and in some cases live independently, with community and social support.

3.2 Moderate Mental Retardation: About $10 \%$ of the mentally retarded population is considered moderate retardation. Moderately retarded individuals have intelligence quotient (IQ) score ranging from 35-55. They can carry out work and self-care tasks with moderate supervision. They typically acquire communication skills in childhood and are able to live and function successfully within community or in a supervised group home.

3.3 Severe Mental Retardation: About 3-4\% of the mentally retarded population is severely retarded. Severe retarded individuals have intelligence quotient (IQ) scores of 20-40. They may master very basic self-care skills and some communication skills. Many severely retarded individuals are able to live in a group home.

3.4 Profound Mental Retardation: Only $1-2 \%$ of the mentally retarded population is classified as profoundly retarded. Profoundly retarded individuals have intelligence quotient (IQ) scores under 20-25. They may be able to develop basic self-care and communication skills appropriate for support and training. Their retardation is often caused by an accompanying neurological disorder. The profoundly retarded need a high level of structure and supervision. The American Association on Mental Retardation (AAMR) has developed another widely accepted diagnostic classification system for mental retardation. The AAMR classification system focuses on the capabilities of the retarded individual rather than on the limitations. The categories describe the level of support required. There are intermittent support, limited support, extensive support, and pervasive support. To 
some extent, the AAMR classification mirrors the DSM-IV classification, intermittent support, for example, is support needed only occasionally, perhaps during times of stress or crisis.

It is the type of support typically required for most mildly retarded individuals. At the other end of the spectrum, pervasive support, or life-long, daily support for most adaptive areas would be required for profoundly retarded individuals.

\section{Causes of Mental Retardation}

Among children, the cause is unknown for one-third to one-half of cases. Generally, there are two major causes of mental retardation namely genetic and environmental causes. The genetic or inherited causes include PKU Disorder, Tay sachs syndrome, fragile X syndrome and Down syndrome. However, Down syndrome, velocariofacial syndrome, and fetal alcohol syndrome are the three most common inborn causes. However, researchers have found many other causes. The most common are genetic conditions are sometimes disability is caused by abnormal genes inherited from parents, errors when genes combine, or other reasons. The most prevalent genetic conditions include Down syndrome, Klinefelter's syndrome, Fragile X syndrome. Neurofibromatosis, congenital hypothyroidism, Williams syndrome, Phenylketonuria (PKU), and Prader-Willi syndrome. Other genetic conditions include: Mowat-Wilson syndrome, genetic ciliopathy, and Siderius type X-linked mental retardation. In the rarest of cases, abnormalities with the $\mathrm{X}$ or -Y chromosome may also cause disability. The 48, XXXX and 49, XXXXX syndrome affects a small number of girls worldwide, while boys may be affected by 47, XYY, 49, XXXXY, or 49, XYYYY.

4.1 Environmental Conditions. The second major cause of mental retardation is environmental hazard. This includes exposure to certain toxins, material illnesses during pregnancy, frequent X-ray during pregnancy, prolonged labour, lack of oxygen etc (Obidigbo, 2007).

4.2 Problems during pregnancy: Mental disability can result when the fetus does not develop properly. For example, there may be a problem with the way the fetus cells divide as it grows. A woman who drinks alcohol or gets an infection like rubella during pregnancy may also have a baby with mental disability (Obidigbo, 2007).

4.3 Problems at birth: If a baby has problems during labour and birth, such as not getting enough oxygen (anorexia), he or she may have developmental disability due to brain damage.

4.4 Exposure to certain types of disease or toxins: Diseases like whooping cough, measles, (German measles) or meningitis can cause mental disability if medical care is delayed or inadequate. Exposure to poisons like lead or mercury may also affect mental ability.

4.5 Iodine deficiency, affecting approximately two billion people worldwide, is the leading preventable cause of mental disability in the developing world where iodine deficiency is endemic. Iodine deficiency also causes goiter, (an enlargement of the thyroid gland). More common than full-fledged cretinism, (as retardation caused by severe iodine deficiency) is mild impairment of intelligence. Certain areas of the world, due to natural deficiency and governmental inaction, are severely affected. India is the most outstanding, with 500 million suffering from deficiency. 54 million from goiter, and 2 million from cretinism. Among other nations affected by iodine deficiency, China and Kazakhstan have instituted widespread iodization (Wells, 2007).

4.6 Malnutrition is a common cause of reduced intelligence in parts of the world affected by famine, such as Ethiopia. 


\section{Diagnosis of Mental Retardation}

According to APA (2005) in their Diagnostic and Statistical Manual of Mental Disorders (DSM-IV), three criteria are required to be met for a diagnosis of mental retardation: namely an IQ below 70, significant limitations in two or more areas of adaptive behaviour measured by an adaptive behaviour rating scale, i.e. communication, self-help skills, interpersonal skills, and more, and evidence that the limitations became apparent before the age of 18. It is formally diagnosed by professional assessment of intelligence and adaptive behaviour.

\subsection{IQ below 70}

The first English-language IQ test, the Terman-Binet, was adapted from an instrument used to measure potential to achieve, developed by Binet in France in 1905. Terman translated the test and employed it as a means to measure intellectual capacity based on oral language, vocabulary, numerical reasoning, memory, motor speed and analysis skills. The mean score on the currently available IQ tests is 100, with a standard deviation of 15 (WAIS/WISC-IV) or 16 (Stanford-Binet). Sub-average intelligence is generally considered to be present when an individual scores two standard deviations below the test mean. Factors other than cognitive ability (depression, anxiety, etc.) can contribute to low IQ scores; it is important for the evaluator to rule them out before concluding that measured IQ is "significantly below average".

The following ranges, based on Standard Scores of intelligence tests, rolled the categories of the American Association of Retardation, the Diagnostic and Statistical Manual of Mental Disorders-IV-TR, and the International Classification of Diseases (Obidigbo, 2009; 2012).

Class

Profound mental retardation

Severe mental retardation

Moderate mental retardation

Mild mental retardation

Borderline intellectual functioning
IQ

$$
\text { below } 20
$$

20-34

$$
35-49
$$$$
\text { 50-69 }
$$

Since the diagnosis is not based only on IQ scores, but must also take into consideration a person's adaptive function, diagnosis is not made rigidly. It encompasses intellectual scores, adaptive functioning scores from an adaptive behaviour scale based on descriptions of known abilities provided by someone familiar with the person, and also the observations assessment examiner who is able to find out directly from the person what he or she can understand and communicate.

\subsection{Significant limitations in two or more areas of adaptive behaviour}

Adaptive behaviour, or adaptive functioning, refers to the skills needed to live independently (or at the minimally acceptable for age). To assess adaptive behavior, professionals compare the functional abilities of a child to those of other children of the same age. To measure adaptive behaviour, professionals use structured interviews, with which they systematically elicit information about persons' functioning in the community from people who know them well. There are many adaptive behaviour scale. Accurate assessment of the quality of someone's adaptive behaviour requires clinical judgment as well. Certain skills are relevant to adaptive behaviour, such as:

- Daily living skills, such as getting dressed, using the bathroom, and feeding oneself.

- Communication skills, such as understanding what is said and being able to answer.

- Social skills with peers, family members, spouses, adults, and others. 


\subsection{Evidence that the limitations became apparent in childhood}

This third condition is used to distinguish mental retardation from dementing conditions such as Alzheimer's disease or acute traumatic injuries with attendant brain damage.

\section{Management of Mental Retardation}

By most definitions mental retardation is more accurately considered as disability rather than a disease. Mental retardation can be distinguished many ways from mental illness, such as schizophrenia or depression based on its ethology and treatment option. Currently, there is no "cure" for mental retardation but early intervention can enrich the intellectual and physical development of people with this condition (Halgin \& Whitebourne, 2005). However, most individuals can learn to do many things with appropriate support and training.

There are thousands of agencies around the world that provide assistance for people with developmental disabilities. This could be exploited by the teacher with a view of letting the child cum family benefit from such services. We have to note also that within one agency there could be departments that include fully residential homes, day-rehabilitation programmes that approximate schools, workshops wherein people with disabilities can get jobs, or be training in special skills like crafts, carpentry and the likes. There are also programmes that assist people with developmental disabilities in obtaining jobs in the community, programmes that provide support for people with developmental disabilities who have their own apartments, programmes that assist them with raising children if they are adults and many more. There are also many agencies and programmes for parents or children with developmental disabilities. Beyond that there are specific programmes that people with developmental disabilities can take part in wherein they learn basic skills. These "goals" may take a much longer amount of time for them to accomplish, but the ultimate goal is independence. This may be anything from independence in tooth brushing to an independent residence. People with developmental disabilities learn throughout their lives and can obtain many new skills even late in life with the help of their teachers, families, caregivers, clinicians and people who coordinate the efforts of all of these people. The teachers may also plan mainstreaming programme for the child. Through the process of mainstreaming people with cognitive and physical disabilities are integrated with non-disable (normal) population. These children are through this method allowed to participate in ordinary classroom where they were provided with the necessary assistance geared to their particular needs.

Although there is no specific medication for mental retardation, many people with developmental disabilities have further complications and may take several medications. For example autistic children with developmental delay may utilize anti-psychotics or mood stabilizers to help with behaviour. This may call for team work with psychiatrist medical doctors, the school and the parents with a view to getting the best treatment for the child. Use of psychotropic medications such as benzodiazepines in people with mental retardation requires monitoring and vigilance as side effects occur commonly and are often misdiagnosed as behavioural and psychiatric problems.

\section{Conclusion}

The paper concludes that since mental retardation is one of the major human development problems of our time and that the consequences of mental retardation in the general population are alarming and devastating, there is need to create adequate awareness especially among school administrators, about this ugly trend. 
The paper posits that three criteria are required to be met for a diagnosis of mental retardation: namely an IQ below 70, significant limitations in two or more areas of adaptive behaviour measured by an adaptive behaviour rating scale, i.e. communication, self-help skills, interpersonal skills, and more, and evidence that the limitations became apparent before the age of 18. It is formally diagnosed by professional assessment of intelligence and adaptive behaviour.

\section{References}

American Psychiatric Association (2005), DSM-IV-TR. Diagnostic and Statistical Manial. Washington, D.C. APAP.

Durand, D. and Barlow, D. (2000). Abnormal Psychology. Canada: Wardsmith.

Halgin, R. and Whiteborne, K. (2005). Abnormal Psychology. New York: McGraw Hill.

Madukonam, E. (1995). Training the mentally retarded children in Nigeria. Paper presented at AIICMHCWCA, Nigerian Chapter. Chumex Press Enugu.

Nwankwo (2001), Abnormal Psychology, Enugu: Ferdinio Publishers.

Obidigbo, G.C.E. (1995). Prospect for Organizing a recognizable training need for MR Children. Paper presented at AIICMHCWCA, Nigerian Chapter. Chumex Press Enugu.

Obidigbo, G.C.E. (2007). Panoramic Issues in Psychology. Enugu: Sages Comm.

Obidigbo, G.C.E. (2009). Handbook of Clinical Psychology. Enugu: Sages Comm.

Obidigbo, G.C.E. (2012). Metal Health and Mental Illness: Never the Twain Shall Meet. Psychology Bulletin (in Press). 